\title{
Identification of a non-purple tartrate-resistant acid phosphatase: an evolutionary link to Ser/Thr protein phosphatases? Kieran S Hadler ${ }^{1}$, Thomas Huber ${ }^{1}$, A Ian Cassady ${ }^{2}$, Jane Weber ${ }^{2}$, Jodie Robinson ${ }^{2}$, Allan Burrows ${ }^{2}$, Gregory Kelly², Luke W Guddat ${ }^{1}$, David A Hume ${ }^{2,3}$, Gerhard Schenk*1 and Jack U Flanagan*3
}

Address: ${ }^{S}$ School of Molecular and Microbial Sciences, The University of Queensland, St. Lucia, 4072, Australia, ${ }^{2}$ Cooperative Research Centre for Chronic Inflammatory Disease, Institute for Molecular Bioscience, The University of Queensland, St. Lucia, 4072, Australia and ${ }^{3}$ ARC Special Research Centre for Functional and Applied Genomics, Institute for Molecular Bioscience, The University of Queensland, St. Lucia, 4072, Australia

Email: Kieran S Hadler - k.hadler@uq.edu.au; Thomas Huber - t.huber@uq.edu.au; A Ian Cassady - i.cassady@imb.uq.edu.au; Jane Weber - j.weber@imb.uq.edu.au; Jodie Robinson - j.robinson@imb.uq.edu.au; Allan Burrows - a.burrows@imb.uq.edu.au; Gregory Kelly - g.kelly@imb.uq.edu.au; Luke W Guddat - luke.guddat@uq.edu.au; David A Hume - d.hume@imb.uq.edu.au; Gerhard Schenk* - schenk@uq.edu.au; Jack U Flanagan* - j.flanagan@auckland.ac.nz

* Corresponding authors

Published: 4 September 2008

BMC Research Notes 2008, I:78 doi:10.1 I86/1756-0500-1-78
Received: 9 June 2008

Accepted: 4 September 2008

This article is available from: http://www.biomedcentral.com/1756-0500/I/78

(c) 2008 Schenk et al; licensee BioMed Central Ltd.

This is an Open Access article distributed under the terms of the Creative Commons Attribution License (http://creativecommons.org/licenses/by/2.0), which permits unrestricted use, distribution, and reproduction in any medium, provided the original work is properly cited.

\begin{abstract}
Background: Tartrate-resistant acid phosphatases (TRAcPs), also known as purple acid phosphatases (PAPs), are a family of binuclear metallohydrolases that have been identified in plants, animals and fungi. The human enzyme is a major histochemical marker for the diagnosis of bonerelated diseases. TRAcPs can occur as a small form possessing only the $\sim 35 \mathrm{kDa}$ catalytic domain, or a larger $\sim 55 \mathrm{kDa}$ form possessing both a catalytic domain and an additional $\mathrm{N}$-terminal domain of unknown function. Due to its role in bone resorption the $35 \mathrm{kDa}$ TRAcP has become a promising target for the development of anti-osteoporotic chemotherapeutics.

Findings: A new human gene product encoding a metallohydrolase distantly related to the $\sim 55$ $\mathrm{kDa}$ plant TRAcP was identified and characterised. The gene product is found in a number of animal species, and is present in all tissues sampled by the RIKEN mouse transcriptome project. Construction of a homology model illustrated that six of the seven metal-coordinating ligands in the active site are identical to that observed in the TRAcP family. However, the tyrosine ligand associated with the charge transfer transition and purple color of TRAcPs is replaced by a histidine.

Conlusion: The gene product identified here may represent an evolutionary link between TRAcPs and Ser/Thr protein phosphatases. Its biological function is currently unknown but is unlikely to be associated with bone metabolism.
\end{abstract}

\section{Background}

Purple acid phosphatases (PAPs) are a diverse group of metalloenzymes that catalyse the hydrolysis of phosphate esters and anhydrides [1]. PAPs are resistant to inhibition by $\mathrm{L}(+)$-tartrate, a potent inhibitor of other acid phosphatases, and as such are also known as tartrate-resistant acid phosphatases (TRAcPs; alternative names include ACP5, TRAP) [1]. They contain a bimetallic active site 
comprising seven coordinating amino acids that are conserved in all PAP isoforms identified to date $[1,2]$. One metal site is invariably an $\mathrm{Fe}$ (III) and the characteristic purple color of TRAcPs arises from a tyrosine to Fe(III) charge transfer transition [1]. The other site contains a divalent metal ion where $\mathrm{M}(\mathrm{II})=\mathrm{Fe}, \mathrm{Zn}$ or $\mathrm{Mn}$ depending on the source of the protein [1-5]. The X-ray crystal structures of TRAcPs from several sources, including human, pig, red kidney bean and sweet potato have been determined [6-9]. Notably, although their sequence identity is only $<20 \%$, these enzymes have a common core structure with five motifs that contain the invariant seven metal coordinating amino acids in the catalytic site [2].

TRAcPs have been isolated from a range of plants, mammals and fungi, and TRAcP-like sequences have also been identified in a number of bacteria [1]. Structural and biochemical characterisation of the TRAcPs from the red kidney bean, Phaseolus vulgaris, and sweet potato, Ipomoea batatas, have demonstrated their existence as homodimers with subunits of $\sim 55 \mathrm{kDa}[1,5]$. The plant isoforms may also exist as heterodimers of 57 and $63 \mathrm{kDa}$ subunits [1]. The catalytic centres of the red kidney bean, soybean and one isoform from sweet potato enzyme contain an $\mathrm{Fe}$ (III)$\mathrm{Zn}$ (II) complex, whereas $\mathrm{Fe}(\mathrm{III})-\mathrm{Mn}$ (II) is present in the other sweet potato form [1]. Plant TRAcPs have been shown to exhibit an amino acid sequence similarity of $>$ $70 \%$ [2]. Mammalian TRAcPs have been characterised from multiple species including human, pig, cow, mouse and rat, and all exist as monomers of $\sim 35 \mathrm{kDa}$, that share $>80 \%$ sequence identity and contain redox-active Fe(III)$\mathrm{Fe}(\mathrm{III}) / \mathrm{Fe}(\mathrm{II})$ centers $[2,10]$. A number of distinct TRAcP isoforms were identified in plants and bacteria, clearly illustrating the existence of multiple TRACP genes in different kingdoms [1,2]. This is further supported by the existence of a plantlike TRAcP in animals [1].

The biological roles for TRAcPs are diverse and speciesdependent. Evidence has accumulated that links the mammalian enzymes to bone metabolism and bacterial killing, while plant enzymes maybe have a function in phosphate metabolism [10]. Specifically, it could be shown that in transgenic mice the level of TRAcP expression correlates with the extent of bone resorption; TRAcPknockout mice display symptoms characteristic for osteoporosis, while mice overexpressing TRAcP display an osteoporotic phenotype $[11,12]$. TRAcP is a major histochemical marker for the diagnosis of bone-related diseases, and elevated serum concentrations of are also observed in patients with Paget's disease, osteosarcoma, breast and prostate cancer. Due to its role in bone resoption TRAcP has become a target for the development of anti-osteoporotic chemotherapeutics [13].
The design of such chemotherapeutics necessitates a high degree of specificity, in particular since enzymes closely related to TRAcPs may function in completely different roles in metabolism. We have thus extended our previous work on investigation of TRAcP and TRAcPlike protein content in animal genomes and identified a new gene product that is a remote homolog to both TRAcPs and Ser/ Thr protein phosphatases.

\section{Findings \\ Homolog identification and characterisation}

The human TRACP (ACP5) sequence (accession number NP_001602; unless stated otherwise accession codes are NCBI reference sequence numbers) was used to perform a five iteration PSI-BLAST search of the non-redundant database (the search conditions were the same as described previously [14]). This search identified a distantly related human sequence with the accession number NP_060810, that had 15\% sequence identity and 29\% similarity to the original acp5 query sequence. Related gene products from other eukaryotes were identified in the NCBI Homologene database http:// www.ncbi.nlm.nih.gov/sites/entrez?db=homologene and ENSEMBL resources http://www.ensembl.org, and included Bos taurus (NP_001026941) Pan troglodytes (XP_001145620), Canis familiaris (XP_536969), Mus musculus (NP_666179), Rattus norvegicus (NP_001013985), Gallus gallus (XP_414732) and Plasmodium falciparum (XP_001348209) indicating that this new gene product is evolutionarily conserved. The new human sequence was used to query the nr database to search for the closest relative with known structure, and identified the catalytic domain of TRAcPs from red kidney bean (P. vulgaris), $4 \mathrm{KBP}$ [6], and sweet potato (I. batatas), 1XZW [8]. Although sequence identities were low (18\% across 246 residues as determined by PSI BLAST analysis) the E-values for the profile based search were $2 \times 10^{-72}$ and $8 \times 10^{-}$ 68 respectively, clearly indicating a significant relationship between these proteins and the novel sequence. Alternative transcripts for the mouse and human sequences were also included.

The sequences identified here were aligned using T-coffee, as shown in Figure 1[15]. As can be seen from the alignment, the sequence conservation across species is high, with the G. gallus gene product $70 \%$ identical to the human, dropping to approximately $32 \%$ for the $P$. falciparum. Illustrated within this figure are five motifs, that are reminiscent of known TRAcP sequences that contain the metal coordinating ligands and can be represented by the patterns (1) DxG, (2) GDx2Y, (3) GNH [E, D], (4) $\mathrm{Vx} 2 \mathrm{H},(5) \mathrm{GHxH}$, where $\mathrm{x}$ represents any amino acid [2]. The notable difference is the concerted substitution of the Tyr in motif 2 by a His in the new sequences. This Tyr is essential for the purple color and the presence of an iron 
Hsa_aTRAcP Ptr_aTRACP Mmu_aTRACP Rno_aTRAcP Cfa_aTRAcP Gga_aTRAcP Pfa_aTRAcP Hsa_TRACP

Hsa_aTRAcP Ptr_aTRAcP Mmu_aTRAcP Rno aTRACP Cfa_aTRAcP Gga_aTRACP Pfa aTRAcP Hsa_TRACP

Hsa_aTRAcP Ptr_aTRAcP Mmu_aTRAcP Rno_aTRAcP Cfa_aTRAcP Gga_aTRAcP Pfa_aTRAcP Hsa_TRACP

Hsa_aTRAcP Ptr_aTRACP Mmu_aTRAcP Rno_aTRAcP Cfa_aTRAcP Gga_aTRAcP Pfa_aTRACP Hsa_TRACP

Hsa_aTRAcP Ptr_aTRACP Mmu aTRACP Rno_aTRAcP Cfa_aTRAcP Gga_aTRACP Pfa_aTRACP Hsa_TRACP

Hsa_aTRACP Ptr_aTRACP Mmu_aTRACP Rno_aTRAcP Cfa_aTRAcP Gga_aTRAcP Pfa_aTRAcP $\mathrm{Hsa}$ TRACP

MSA-AEAGGVFHRARGRTLDAFPAEKESEWKGPFYF ILGADP QFGLIKAWSTGDCDNGGD MSA-AEAGGVF HRARGRTLDAFPAEKESEWKGP FYF I LGADP QF GLMKAWS T GDCDNGGD MSA-MEAADVFHRARGRTLDAFSSEKEREWKGPFYFVQGADTQFGLMKAWSTGNCDAGGD MSA-AGAADVF HRARGRTLDAF S SEKEREWTGPFYFVQGADP QF GLMKAWS T GNCDNGGD MSA-AEAGGVFRRARGRTLDAFP SEKEREWKGPFYF IQGADP QF GLMKAWSTGD SDSGGD MSVMAAVGDVFRRARGRTLTAFRQGDEYQWKGPFYF I QGADP QF GLIKAWAVGNTGSGDD -----MVLIKVENHEGKNVNANKFE-------PFFEVLFGD IQYGMIR---------GNH

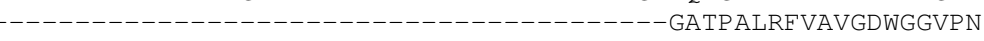
SSSSSS

EWEQEIRLTEQAVQA INELNP -KP KFFVLCGDLI HAMP GKPWRTEQTEDLKRVLRAVDRA EWEQEIRLTEQAVQA INKLNP -KP KFFVLCGDL I HAMP GKPWRTEQTEDLKRVLRAVDRA EWGQE IRLTEQAVEA INKLNP - KP KFFVLCGDLVHAMP GTPWRQEQTRDLQRVLKAVDQD EWGQEIRLTEQAVEA INKLNP - KP KFFVLCGDLVHAMP GTRWRKEQTRDLQRVLKVVDQD EWGQEIRLTEQAVQA INKLKP -KPRFFVLCGDL I HAMP GMP WRKEQTADLQRVLTQVDSD EWGEE IKLTEQAVQA INKLNP - KP KF FVLCGDL I HGMP GTQWRKDQEQDLKNVLKNTDQD GWYEERELLKSAIEKTNKL---KPP FVVALGDLTNKFP LDP IQTNQITDLKNDFKLLDKD AP FHTAREMANAKEIARTVQILGADF ILSLGDNFYFTGVQD INDKRFQETFEDVF SDRSL

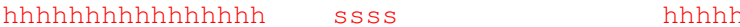

IPL--VLVSGNHD IGNTP TAETVEEFCRTWGDDYFSFWVGGVLFLVLNSQFYENP SKCP S IPL--VLVSGNHD I GNAP TAETVEEFCRTWGDDYF SFWVGGVLFLVLNSQFYENP SKCP S IPL--VMVSGNHDLGNAP TAETVEEFCQTWGDDYF SFWVGGVLF LVLNSQF LYDASRCPA IPL--VLVSGNHDLGNAP TAETVEEFCQTWGDDYF SFWVGGALFLVLNSQF LYDASKCPA IPL--VLVSGNHDVGNTPTPETVAEFQQTWGDDYF SFWVGGVLCLVLNSOFWYDASRCPA IPL--VFVSGNHD I GNTP TRET IDNYCKSWGDDYF SFWVGGVFF LVLNSQLYFDSSKCPE IDL--YVFCGNHDVGNKP SMEGMEYFEEQWGD SYYSFVYNNCAF IVLNSP I LYDETHVKE RKVPWYVLAGNHDHLGNVSAQIAYSKISKRWNFP SPFYRLHFKIPQTNVSVAIFMLDTVT

$$
\text { SSS hhh hhhhhhhhh SS SSSSS SSSSS hhh }
$$

LKQAQDQWLDEQ---------------LS IARQRH-CQHA IVFQH IP LF LES IDEDDDY LKQAQDQWLDEQ---------------LS IARQRH-CQHA IVFQH IP LF LES IDEDDDY LKQAQDHWLDQQ---------------LNIAEQKQ-CQHAIVFQHIP LF LQS IDEDDDLKQAQDHWLDQQ---------------LS IAEQQQ-CQHA IVF $Q H$ IP LF LKS IDEDDD-

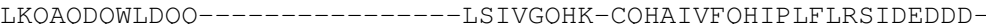
LKQAQDAWLNGQ---------------LAAAEKRK-CKHI IVFQH IP LF LRKP DEDHDMKEEQLKWLEKT----------------LEKLHSLN-VKHKF LLLHHALMYDD I YEGENI LCGNSDDF LSQQPERPRDVKLARTQLSWLKKQLAAAREDYVLVAGHYPVWS IAEHGPT--

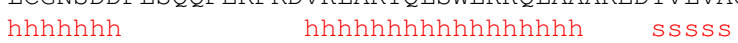

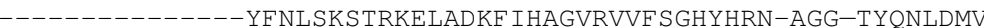
$-------------Y F N L S K S T R K K L A D K F I H A G V K V V F$ S GHYHRN-AGG-TYQNLDMV --------------YFNLTKTVRKELAFKLTRAGIRAVFSGHYHRN-AGG-TYONLDMV $-------------Y F N L T K T V R Q E L A D K F T R A G I R A V F$ SGHYHRN-AGG-TYQNLDMV $------------Y F N L T K S V R K E$ IANKLAGAGVTAVF SGHYHRN-AGG-TYQNLDMV YFNLEKSVRQE IMEKFQNAGVKAVFSGHYHRN-AGG-WYRGLEMV GLIYGDKFHNYSEKNEFHLKKEPRLF IYELMKKYKVTHVFCAHLHANREND-IDHNIKQI ------------------HCLVKQLRP LLATYGVTAYLCGHDHNLQYLQ-DENGVGYV

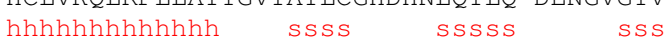

VSSAIGCOLGRDP HGLRVVVVTAEKIVHRYYSLDELSEKGIEDDLMDLIKK-K VSSAIGCQLGRDPHGLRVVVVTAEKIVHRYYSLDELSEKGIEDDVMDLIKK-K VSSAIGCQLGKDTHGLRVVAITAEKIVHRYYSLDELSQGGVEEDLKELLK--E VSSAIGCQLGKDTHGLRVVVVTAEKIVHRYYSLDELSKRGLDDDLRELLK--E VSSAIGCQLGQDTHGLRVVVVTAKKIVHRYYSLDELSEKGIEDDLMGLMKE-K VSSAIGCQLGEDKHGLRVVLVTDEKIVHRYYSLDELSSQGLEKEMVDMLAKQN TISAVGMQAKDDKSGIFIVQVTEDKVDYKYYPFEYPFFFFFFEHGS------LSGAGNFMDP SKRHQRKVPNGYLRFHYGTEDSLGGFAYVEISSKEMTVTYIEA

$$
\text { SS hhh SSSS SSSSS SSSSSS }
$$

\section{Figure I}

Multiple sequence alignment of the eukaryotic aTRACP gene products. Alternative transcripts identified in mouse and human are appended. The five motifs containing the seven metal coordinating residues observed in TRAcPs have been superimposed on the alignment (Me_motif_IXZW). The position is based on the WURST generated pairwise alignment between the human gene product and the sweet potato enzyme. For comparison the sequence of human TRAcP (Hsa_TRAcP) is also included, together with its secondary structure elements (h: helix; s: $\beta$-sheet; sheet 14 is omitted). Species identifiers: Hsa, Homo sapiens; Ptr, Pan troglodytes; Mmu, Mus musculus; Rno, Rattus norvegicus; Cfa, Canis familiaris; Bta, Bos Taurus; Gga, Gallus gallus; Pfa, Plasmodium falciparum. 
in the trivalent oxidation state in the active site of TRAcPs (see above), whereas a His residue in this position is seen in other binuclear metallohydrolases including the Ser/ Thr protein phosphatases [1]. Based on the identity of the amino acid residues that are likely to line the active site pocket in the novel gene product it is probably that this protein is a non-purple enzyme with phosphatase activity. We have thus labelled it $H s a \_$aTRAcP (aTRAcP: alternative TRAcP).

A large number of phosphatases are present in eukaryotic organisms. Many acid phosphatases, including the mammalian TRAcPs, are lysosomal enzymes and have signal peptides and lysosomal targeting sequences. No such sequences are evident in this new protein. To test the location within the cell, we constructed a mammalian expression plasmid with a V5 epitope tag (Figure 2). When this tagged protein is expressed in RAW264 macrophages, the predominant location is diffuse cytoplasmic with no evident membrane association. It is thus likely that the biological role of $H s a \_$aTRAcP is different from that of its purple counterparts.

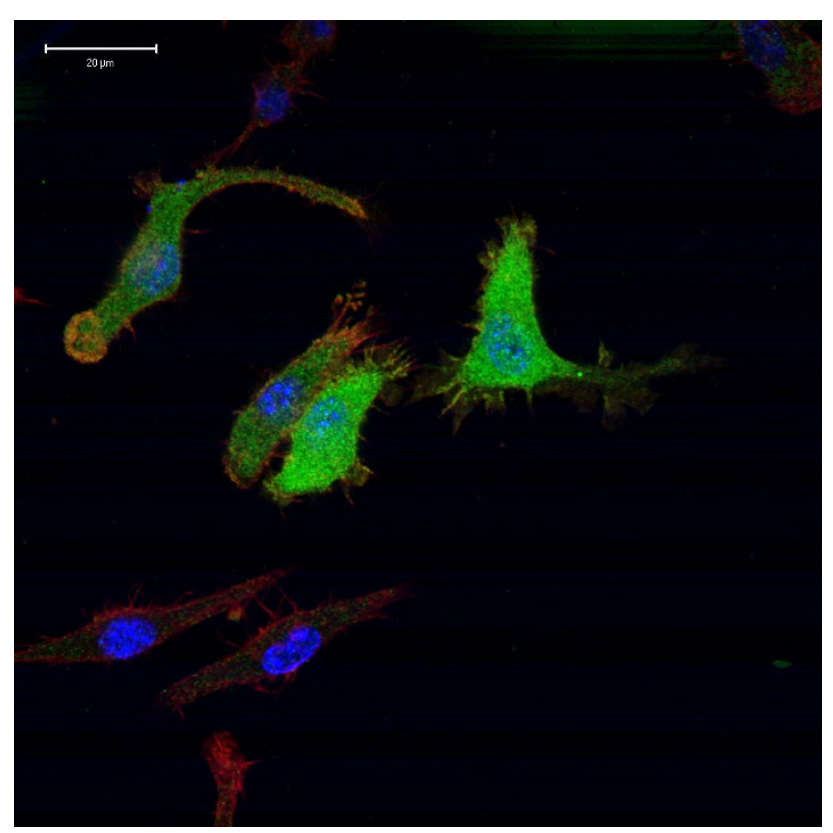

Figure 2

Subcellular localisation of Hsa_aTRACP. Immunofluoresence staining of RAW264.7 cells transfected with a Hsa_aTRACPV5 using the Alexa488 goat anti-mouse antibody (Invitrogen) in combination with the mouse anti-V5 $\lg G 2 a$ (Serotec) and visualised using Alexa594 Phalloidin stain (Invitrogen). DNA was stained with DAPI (Roche). Evident is the diffuse cytoplasmic distribution of Hsa_aTRACP.
The intron-exon structure of the gene encoding this putative phosphatase (C530044N13Rik; ENSEMBL Gene ENSMUSG00000065979) comprises only 4 exons, spread over more than $100 \mathrm{~kb}$ of genomic DNA, a structure that is widely conserved in vertebrates. From analysis of RIKEN transcriptome data for the mouse homolog (GeneID 223978) using the CAGE analysis viewer http://fan tom.gsc.riken.go.jp/, it is evident that the gene locus is actively transcribed in almost all tissues examined, including embryonic tissue as well as adult liver, lung, macrophages and neural tissue with little variation in CAGE Tag frequency (an index of gene expression). The promoter is conserved between mice and human, is relatively GC-rich, and initiates transcription at multiple sites in a $100 \mathrm{bp}$ window around the site of the largest CAGE tag cluster, features consistent with a possible "housekeeping" gene function.

\section{Structure prediction of Hsa_aTRAcP}

To further assess the novel sequences as non-purple binuclear metallohydrolases, a structural model of $H s a \_$aTRAcP was constructed by comparative modelling using the sweet potato TRAcP coordinates [8]. The only proteins with known structure identified from the PSIBLAST search were the plant TRAcPs and the phosphodiesterase from Mycobacterium tuberculosis (Rv0805, 2HY1 [16]). An additional phosphodiesterase was identified from Enterobacter aerogenes (2dxn [17]), using the threading based approach mGenThreader [18]. The prediction reliability scores for the bacterial diesterases were 114.5 and 105.8, respectively, with corresponding p-values (probabilities of false positives) of $1 \times 10^{-10}$ and $1 \times 10^{-9}$. These values are similar to those obtained for the closest TRACP homologue, the enzyme from sweet potato (1XZW, with a reliability score of 87.3 and a p-value of 8 $\times 10^{-8}$. This strongly implies that $H s a \_a T R A c P$ will adopt a fold similar to these proteins.

Due to the low sequence identity between the $H s a \_$aTRACP and the sweet potato enzyme, the sequenceto-structure alignment method WURST was used to generate an alignment (Figure 3), that was subsequently used as input into MODELLER for coordinate generation.

In general, TRAcPs typically consist of two $\beta$-sheets each with seven strands and flanked by two $\alpha$-helices, and is illustrated in Figure 4A for both the plant template structure, and the smaller mammalian isoform. Five of the beta strands position loop structures that contain metal coordinating ligands [7]. Inspection of the model indicates that $H s a \_a T R A C P$ has both sheets conserved, however, one has only six strands and the second, three (Figure 4A and $4 \mathrm{~B}$ ). Notably, the strands contributing the metal coordinating ligands are all conserved in $\mathrm{Hsa}_{-}$aTRACP. Furthermore, loops contributing to substrate binding in 


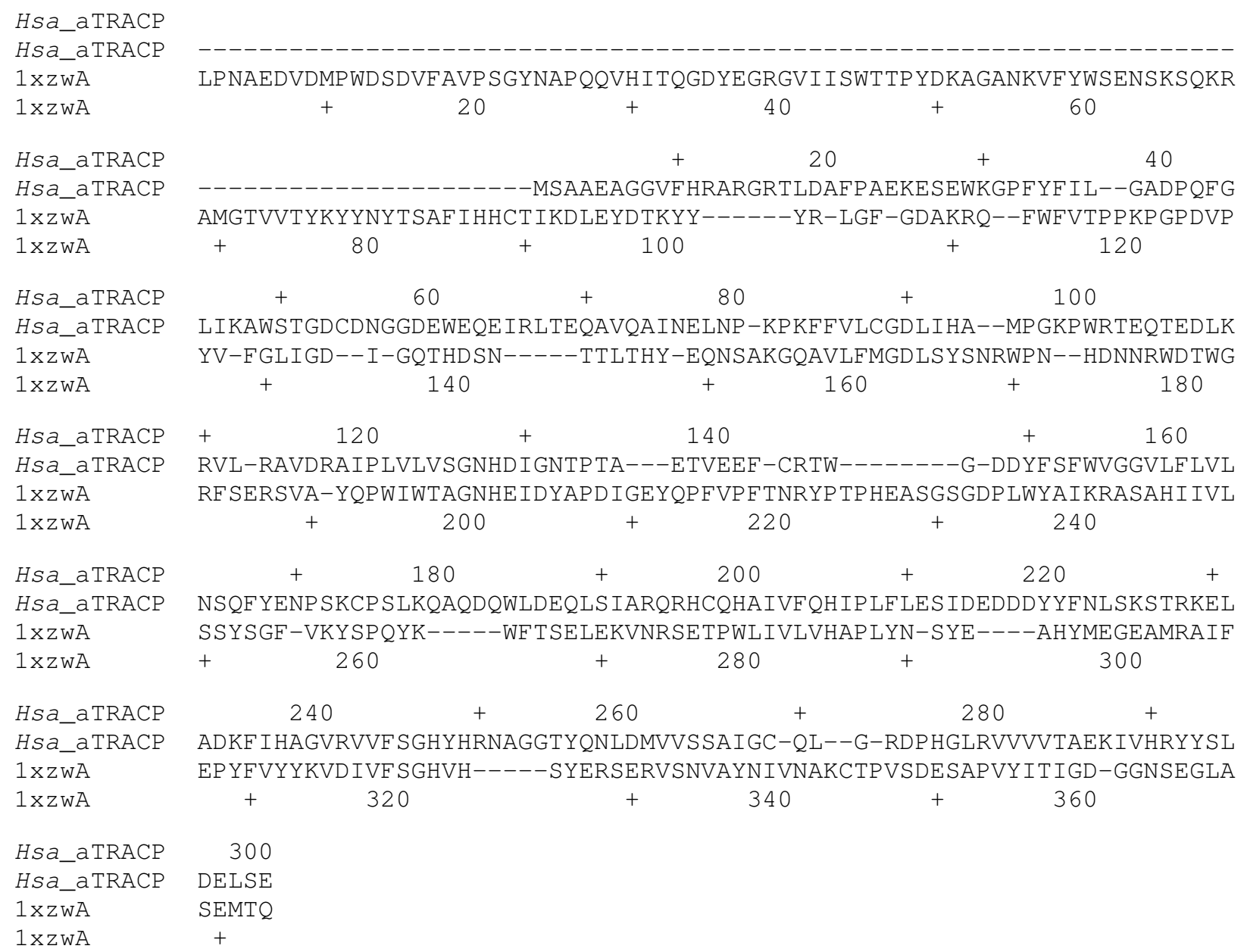

Figure 3

Sequence to structure alignment generated by WURST for the query sequence, Hsa_aTRACP (Accession number Hsa_NP_0608I0), and the template structure from sweet potato TRAcP, pdb code IXZW.

TRACPs and TRACP-like proteins are partially conserved in our model structure. The I-TASSER server was used to produce alternative predicted structures, and gave a top ranked model that appeared to be a composite of the plant PAP and bacterial Rv0805 structures, and that has a confidence score of 0.24 [19]. This model predicted that the second sheet may have two additional strands, similar to the sheet composition of the bacterial enzyme, while the active site loop conformations were highly similar to the plant TRACP rather than the bacterial metallohydrolase.

The model of Hsa_aTRACP reveals that the side chains of seven metal coordinating residues are likely to be spatially conserved in comparison to other binuclear metallohydrolases. The identity of six of the seven residues in Hsa_aTRACP are identical to that in TRAcPs with the exception that Tyr166 (sweet potato TRAcP numbering) is replaced by His93 in $\mathrm{Hsa}_{\text {aTRACP }}$ (Figure 5). Closer inspection of the model indicates that although Tyr219, 220 and 292 are located within a putative substrate binding site, they are not likely to form a charge transfer interaction with the metal ions in the active site. This places $H s a$ aTRACP into a separate, nonpurple class of binuclear metallohydrolases with two soft metal binding sites that are likely favour the coordination of two divalent metal ions. A similar active site structure was reported for other members of the binuclear metallohydrolase family, notably Ser/Thr type protein phosphatases (PPs) such as the ones from bacteriophage $\lambda(\lambda P P)[20]$ and several mammalian organisms, i.e. one from rabbit (PP1) $[21,22]$ and cow (PP2B) [23] and two from human (PP2B and PP5) [24], and more recently the Rv0805 cyclic nucleotide phosphodiesterase from M. tuberculosis [16]. PP2B is also 

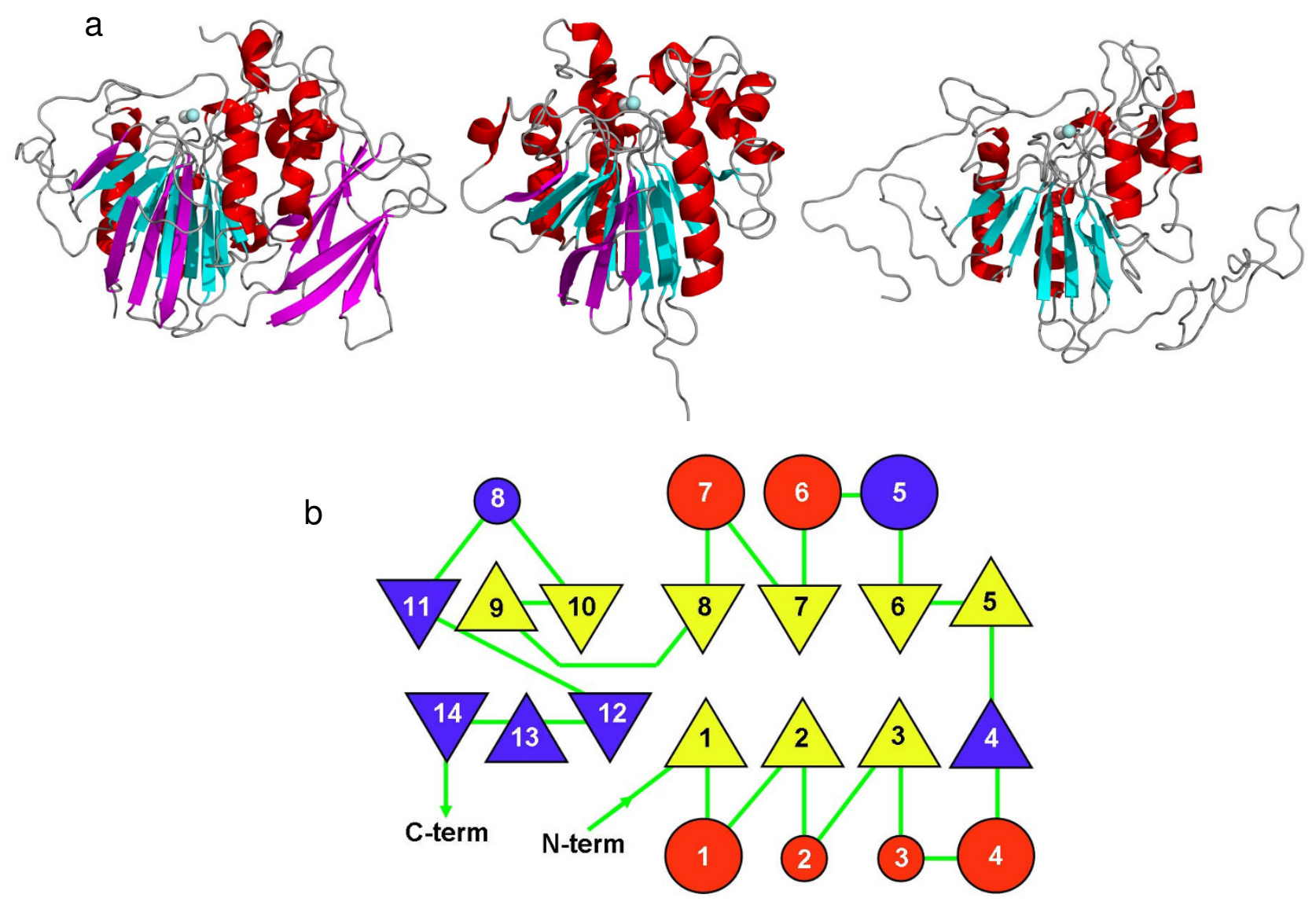

\section{Figure 4}

(A) Cartoon diagrams of the high molecular weight sweet potato TRAcP template structure, IXZW, the low molecular weight human TRAcP (Acp5), IWAR, and the query sequence, Hsa_aTRACP. Secondary structure elements not shared between the known structures and Hsa_aTRACP model are colored magenta. The Fe(III) Fe(II) atoms in the active site are represented as spheres. (B) Comparison of topologies for the low molecular weight human TRAcP and Hsa_aTRACP. Secondary structure elements that are common to both proteins are colored yellow (for $\beta$-strands) and red for ( $\alpha$-helices). Secondary structure regions that are only observed in human TRAcP are colored blue. For Hsa_aTRAcP the regions of secondary structureare SI(residues 49-5I), HI (67-74), S2(85-87), H2(I02-I I0), S3(I2I-I22), H3(I27-I30), H4(I4I-I44), S5(I5I-I 55), S6(I58I62), H6(I80-191), S7(199-203), H7(225-238), S8(242-245), S9(256-258) and SI0(280-284). For human TRAcP

(Hsa_TRAcP; see also Fig. I) the regions of secondary structure are SI(5-I0), HI (24-39), S2(44-47), H2(64-68), S3(83-85), H3(9I-93), H4(96-I04), S4(I09-I I0), S5(I I6-I 2 I), S6(I 28-I33), H5(I36-I45), H6(I57-I 73), S7(I 78-I 82), H7(I 97-209), S8(2I4-2I7), S9(223-227), SIO(233-237), H8(250-252), SI I(258-262), SI2 (270-276), SI3 (280-287) and SI4(292-299).

known as calcineurin and plays a major role in the signal transduction cascade in T-cell activation [25]. The in vivo metal ion contents of PPs is not certain, but all are reported as $\mathrm{M}(\mathrm{II})-\mathrm{M}(\mathrm{II})$ forms, where $\mathrm{M}=\mathrm{Fe}$, $\mathrm{Zn}$ or $\mathrm{Mn}$ [1].

\section{Conclusion}

Previously we identified a high molecular weight human TRAcP [14]. Here we have extended this study through the characterisation of a second transcript, $H s a$ aTRACP, that is a remote relative of the PAPs, sharing $18 \%$ sequence identity with the plant enzymes as the closest relatives with known structure. Analysis of the active site of Hsa_aTRACP indicates that it is not likely to be a purple protein due to the absence of an essential tyrosine ligand (Figure 5). In this respect, Hsa_aTRACP resembles some cyclic nucleotide phosphodiesterases and novel Ser/Thr PPs. This may therefore represent an event of divergent evolution in the binuclear metallohydrolase family. Based upon the pattern of expression and putative cytoplasmic location, we speculate that Hsa_aTRACP is another member of the cytoplasmic protein phosphatase family that is likely to have a role in the regulation of signalling. 


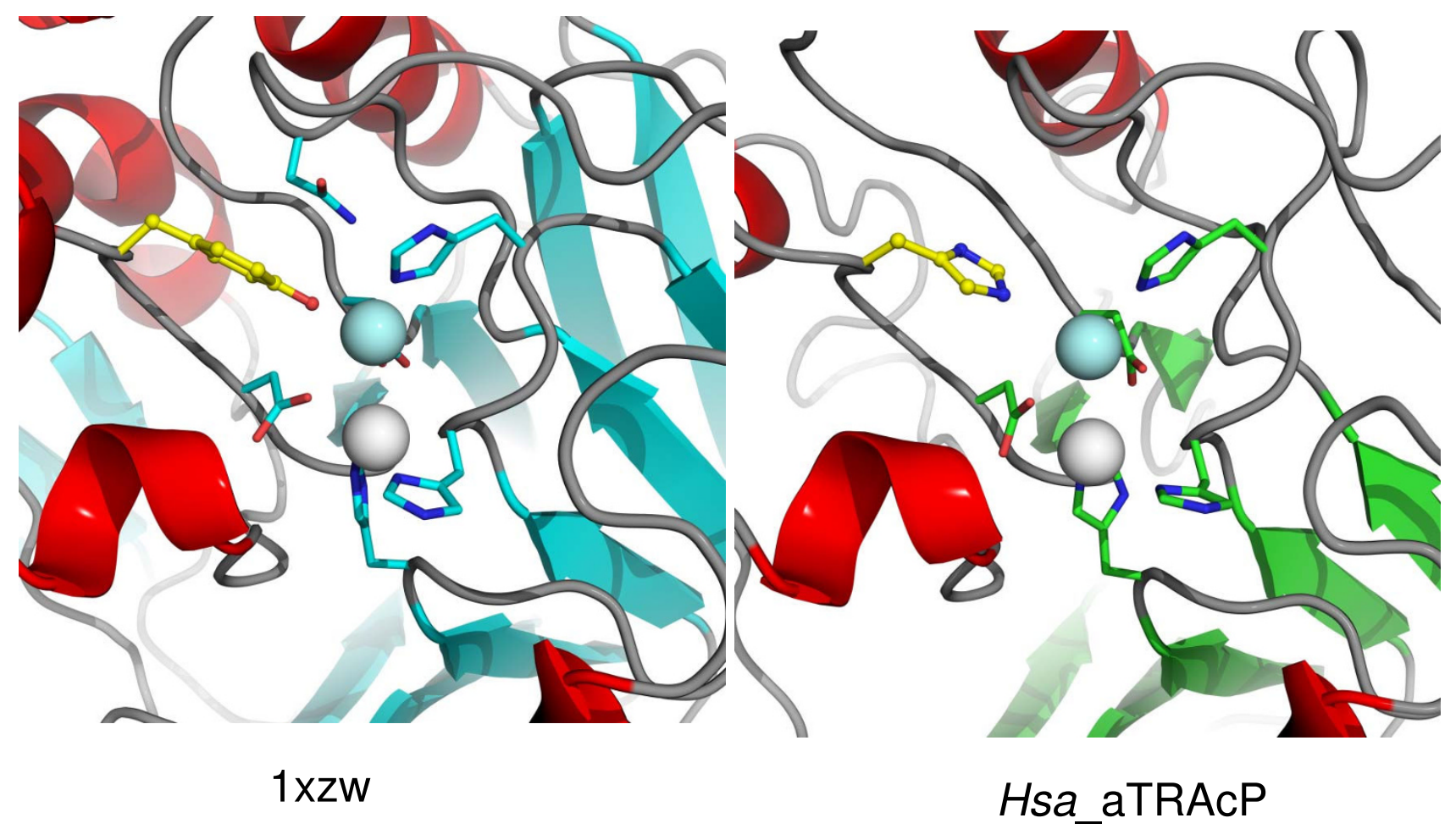

Figure 5

Structure of the modelled active site of Hsa_aTRAcP and that of IXZW illustrating the difference in the Fe(III) coordination site. Substitution of the sweet potato TRAcP Tyr for His in Hsa_aTRACP (yellow, ball and stick representation) indicates that Hsa_aTRACP is not a member of the TRAcP family as the charge transfer transition required for the purple color is absent.

\section{Abbreviations}

PAP: purple acid phosphatase; PP: Ser/Thr protein phosphatase; TRAcP: tartrate-resistant acid phosphatase; rmsd: root mean square deviation.

\section{Competing interests}

The authors declare that they have no competing interests.

\section{Authors' contributions}

$\mathrm{KSH}$ generated homology model, performed database searches and contributed to manuscript writing. TH performed structure alignments. IC, LWG and DAH were interpreting data, designed experiments and provided critical feedback on the manuscript. JW, JR, AB and GK carried out cloning and expression experiments. GS and JUF were responsible for the conceptualization of experiments, analysis and interpretation of data, and drafting and critical review of manuscript.

\section{Acknowledgements}

This work was funded by a grant from the Australian Research Council (DP0558652).

\section{References}

I. Mitić N, Smith SJ, Neves A, Guddat LW, Gahan LR, Schenk G: The catalytic mechanisms of binuclear metallohydrolases. Chem Rev 2006, 106:3338-3363.

2. Schenk G, Guddat LW, Ge Y, Carrington LE, Hume DA, Hamilton S, de Jersey J: Identification of mammalian-like purple acid phosphatases in a wide range of plants. Gene 2000, 250:1 I7-125.

3. del Pozo JC, Allona I, Rubio V, Leyva A, de la Pena A, Aragoncillo C, Paz-Ares ]: A type 5 acid phosphatase gene from Arabidopsis thaliana is induced by phosphate starvation and by some other types of phosphate mobilising/oxidative stress conditions. Plant J 1999, 19:579-589.

4. Li D, Zhu H, Liu K, Liu X, Leggewie G, Udvardi M, Wang D: Purple acid phosphatases of Arabidopsis thaliana. Comparative analysis and differential regulation by phosphate deprivation. J Biol Chem 2002, 277:27772-2778I.

5. Schenk G, Ge Y, Carrington LE, Wynne CJ, Searle IR, Carroll BJ, Hamilton S, de Jersey J: Binuclear metal centers in plant purple acid phosphatases. Fe-Mn in sweet potato and $\mathrm{Fe}-\mathrm{Zn}$ in soybean. Arch Biochem Biophys 1999, 370:183-189.

6. Sträter N, Klabunde T, Tucker P, Witzel H, Krebs B: Crystal structure of a purple acid phosphatase containing a dinuclear Fe(III)-Zn(II) active site. Science 1995, 268: | 489- I 492.

7. Guddat LW, McAlpine AS, Hume D, Hamilton S, de Jersey J, Martin $\mathrm{JL}$ : Crystal structure of mammalian purple acid phosphatase. Structure 1999, 7:757-767.

8. Schenk G, Gahan LR, Carrington LE, Mitić N, Valizadeh M, Hamilton SE, de Jersey J, Guddat LW: Phosphate forms an unusual tripodal complex with the Fe-Mn center of sweet potato purple acid phosphatase. Proc Natl Acad Sci USA 2005, 102:273-278.

9. Sträter N, Jasper B, Scholte M, Krebs B, Duff AP, Langley DB, Han R, Averill BA, Freeman HC, Guss M: Crystal structures of recombinant human purple acid phosphatase with and without an 
inhibitory conformation of the repression loop. J Mol Biol 2005, 35 I:233-246.

10. Mitić N, Valizadeh M, Leung EWW, de Jersey J, Hamilton S, Hume DA, Cassady Al, Schenk G: Human tartrate-resistant acid phosphatase becomes an effective ATPase upon proteolytic activation. Arch Biochem Biophys 2005, 439:154-164.

II. Angel NZ, Walsh N, Forwood MR, Ostrowski MC, Cassady Al, Hume $D A:$ Transgenic mice overexpressing tartrate-resistant acid phosphatase exhibit an increased rate of bone turnover. I Bone Mineral Res 2000, 15:103-I I0.

12. Hayman AR, Jones SJ, Boyde A, Foster D, Colledge WH, Carlton MB, Cox TM: Mice lacking tartrate-resistant acidphosphatase (Acp5) have disrupted endochondral ossification and mild osteoporosis. Development 1996, I 22:3151-3162.

13. Valizadeh M, Schenk G, Nash K, Oddie GW, Guddat LW, Hume DA, de Jersey J, Burke TR, Hamilton S: Phosphotyrosyl peptides and analogues as substrates and inhibitors of purple acid phosphatases. Arch Biochem Biophys 2004, 424:154-162.

14. Flanagan JU, Cassady Al, Schenk G, Guddat LW, Hume DA: Identification and molecular modeling of a novel, plant-like, human purple acid phosphatase. Gene 2006, 377:12-20.

15. Notredame C, Higgins DG, Heringa J: T-Coffee: A novel method for fast and accurate multiple sequence alignment. J Mol Biol 2000, 302:205-217.

16. Shenoy AR, Capuder M, Draskovic P, Lamba D, Visweswariah SS, Podobnik M: Structural and Biochemical Analysis of the Rv0805 cyclic nucleotide phosphodiesterase from Mycobacterium tuberculosis. J Mol Biol 2007, 365:2 II-225.

17. Jackson CJ, Carr PD, Liu J-W, Watt SJ, Beck JL, Ollis DL: The structure and function of a novel glycerophosphodiesterase from Enterobacter aerogenes. J Mol Biol 2007, 367:1047-1062.

18. McGuffin LJ, Jones DT: Improvement of the GenTHREADER method for genomic fold recognition. Bioinformatics 2003, 19:874-88|.

19. Zhang Y: I-TASSER server for protein 3D structure prediction. BMC Bioinform 2007, 9:40.

20. Voegtli WC, White DJ, Reiter NJ, Rusnak F, Rosenzweig AC: Structure of the bacteriophage $\lambda$ Ser/Thr protein phosphatase with sulfate ion bound in two coordination modes. Biochemistry 2000, 39:15365-15374.

21. Goldberg J, Huang HB, Kwon YG, Greengard P, Nairn AC, Kuriyan J: Three dimensional structure of the catalytic subunit of protein serine/threonine phosphatase-I. Nature 1995, 376:745-753.

22. Egloff M-P, Cohen PTW, Reinemer P, Barford D: Crystal structure of the catalytic subunit of human protein phosphatase $I$ and its complex with tungstate. J Mol Biol I 995, 254:942-959.

23. Griffith JP, Kim JL, Kim EE, Sintchak MD, Thomson JA, Fitzgibbon MJ, Fleming MA, Caron PR, Hsiao K, Navia MA: X-ray structure of calcineurin inhibited by the immunophilin-immunosuppressant FKBP I 2-FK506 complex. Cell 1995, 82:507-522.

24. Kissinger CR, Parge HE, Knighton DR, Lewis CT, Pelletier LA, Tempczyk A, Kalish VJ, Tucker KD, Showalter RE: Crystal structures of human calcineurin and the human FKBPI2-FK506-calcineurin complex. Nature 1995, 378:64I-644.

25. Clipstone NA, Crabtree GR: Identification of calcineurin as a key signalling enzyme in T-lymphocyte activation. Nature 1992, 357:695-697. http.//www.biomedcentral.com/1756-0500/1/78 